Irish Math. Soc. Bulletin

Number 76, Winter 2015, 29-30

ISSN 0791-5578

\title{
TOPICS IN COCYCLIC DEVELOPMENT OF PAIRWISE COMBINATORIAL DESIGNS
}

\author{
RONAN EGAN
}

This is an abstract of the PhD thesis Topics in Cocyclic Development of Pairwise Combinatorial Designs written by Ronan Egan under the supervision of Dane Flannery at the School of Mathematics, Statistics, and Applied Mathematics, National University of Ireland, Galway and submitted in July 2015. This thesis is a compilation of results dealing with cocyclic development of pairwise combinatorial designs.

Motivated by a classification of the indexing and extension groups of the Paley Hadamard matrices due to de Launey and Stafford, we investigate cocyclic development of the so-called generalized Sylvester (or Drake) Hadamard matrices. We describe the automorphism groups and derive strict conditions on possible indexing groups, addressing research problems of de Launey and Flannery in doing so.

The shift action, discovered by Horadam, is a certain action of any finite group on the set of its 2-cocycles with trivial coefficients, which preserves both cohomological equivalence and orthogonality. We answer questions posed by Horadam about the shift action, in particular regarding its fixed points. One of our main innovations is the concept of linear shift representation. We give an algorithm for calculating the matrix group representation of a shift action, which enables us to compute with the action in a natural setting. We prove detailed results on reducibility, and discuss the outcomes of some computational experiments, including searches for orthogonal cocycles.

Using the algorithms developed for shift representations, and other methods, we classify up to equivalence all cocyclic $\mathrm{BH}(n, p)$ s where $p$ is an odd prime (necessarily dividing $n$ ) and $n p \leq 100$. This was

2010 Mathematics Subject Classification. 05B20, 20B25, 20 J06.

Key words and phrases. Cocyclic development, generalized Hadamard matrix.

Received on 14-12-2015.

Support from the Irish Research Council and an NUI Galway Hardiman scholarship is gratefully acknowledged. 
achievable with the further aid of our new non-existence results for a wide range of orders.

School of Mathematics, Statistics and Applied Mathematics, NaTiONAL University of Ireland, Galway

E-mail address, R. Egan: r.egan3@nuigalway.ie 